\title{
COMPARATIVE INVESTIGATION OF VLM CODES FOR JOINED-WING
}

\section{ANALYSIS}

\author{
Jordan Hadjiev $^{1}$, Hristian Panayotov ${ }^{2}$ \\ ${ }^{1}$ Doctoral student, ${ }^{2}$ Assoc. professor, TATT, Technical University-Sofia, Branch Plovdiv \\ thugsoulja@abv.bg,hristian@tu-plovdiv.bg
}

\begin{abstract}
The Vortex lattice method (VLM) is a popular aerodynamic analysis method used at the early stages of aircraft design. In this paper a comparative investigation of several computer programs applying the VLM is carried out and their ability to simulate non conventional wing configurations is evaluated. It was found that Athena Vortex Lattice (AVL) code calculated the most correct results, but other programs like Tornado provide useful supplemental information.
\end{abstract}

Keywords: Vortex lattice method, closed wing, joined wing

\section{INTRODUCTION}

The ever increasing requirements for noise and emissions reduction and the need to cut the direct operative costs for transport aircraft are demanding the exploration of new aircraft configurations, because conventional aircraft are no longer able to meet the new requirements. For a large transport aircraft during cruise flight, about $43 \%$ of total drag is due to induced drag. Induced drag depends on the lift distribution along the wing span which, for today's large transport aircraft, is optimized to the extent that no further significant induced drag reduction is possible. In order further improvements to be achieved, non-conventional, non-planar wing configurations should be applied. Closed wing configurations of the boxwing type promise the biggest reduction in induced drag. They are able to provide a lot of other advantages as well, as pointed in [1]. However the closed wing system represents a much more complex subject for aerodynamic analysis and optimization than the conventional cantilever wing. It is characterized by very strong aerostructural coupling. In order the promised advantages of this configuration to be realized the application of multidisciplinary design optimization (MDO) is a must [2]. This makes the selection of an efficient aerodynamic analysis tool very important. It should balance between sufficient accuracy and computational cost. This is especially true in the conceptual design stage, when the basic aircraft geometry should be defined and the whole design space should be explored quickly and efficiently.

The Vortex lattice method, (VLM), is a numerical, computational aerodynamics method that finds broad use. Like the panel methods VLM is based on the potential flow theory and the solution of Laplace's Equation:

$$
\nabla^{2} \phi=0
$$

This equation is exact when the flow is incompressible, inviscid and irrotational. The incompressible potential flow model provides reliable predictions over a wide range of conditions. For the potential flow assumption to be valid for aerodynamics calculations, the viscous effects must be small and the flowfield must be subsonic everywhere. Subsonic compressible flow can be modeled if the Prandl-Glauert transformation is applied.

The key feature of Laplace's equation is the property that allows the equation governing the flowfield to be converted from a $3 \mathrm{D}$ problem throughout the field to a $2 \mathrm{D}$ problem for finding the potential on the surface. The solution is then found by distributing "singularities" of unknown strength over discretized parts of the surface. The strengths of the singularities are determined by solving a linear set of algebraic equations. The VLM is different from the panel methods in that additional conditions are imposed:

- the lifting surfaces are thin. The influence of thickness on aerodynamic forces is neglected;

- boundary conditions are applied on a mean surface, not the actual surface;

- the angle of attack and the angle of sideslip are both small.

Despite the number of assumptions the VLM provides remarkable insight into wing aerodynamics and component interaction. The VLM cannot compute the viscous drag, but the induced drag can be reliably estimated. It provides useful information required to design the control system and to define the load distributions for structural design.

The VLM has been applied for the investigation of joinedwing aircraft $[3,4]$. 
There are many ways to solve the problem and many competing codes. Differences in approaches to the implementation include the use of various singularities.

The purpose of this paper is to compare two popular VLM codes and a program implementing the Blade element method (BEM) in their ability to simulate the properties of a closedwing aircraft.

\section{AVL (Athena Vortex Lattice) [5]}

AVL is a program for the aerodynamic and flight-dynamic analysis of rigid aircraft of arbitrary configuration. It employs an extended vortex lattice model for the lifting surfaces, together with a slender-body model for fuselages and nacelles. General nonlinear flight states can be specified. The flight dynamic analysis combines a full linearization of the aerodynamic model about any flight state, together with specified mass properties.

\section{Tornado [6]}

Tornado is a Vortex Lattice Method for linear aerodynamic wing design applications in conceptual aircraft design or in aeronautical education. By modeling all lifting surfaces as thin plates, Tornado can solve for most aerodynamic derivatives for a wide range of aircraft geometries. The code is implemented in MATLAB and is being used at many universities and corporations around the world.

\section{X-Plane}

$\mathrm{X}$-Plane is a commercial product for flight simulations with huge capabilities. X-Plane is not a game, but an engineering tool that can be used to predict the flying qualities of fixedand rotary-wing aircraft with incredible accuracy. One of its modules, the Plane Maker allows us to model an aircraft with custom designed geometry and see how it performs in flight. $\mathrm{X}$-Plane uses the Blade Element Theory (BEM) to determine the aerodynamic forces for the flight simulation.

\section{PRODUCTS SOLVER CAPABILITIES}

\section{AVL:}

- Solving singularities - Horseshoe vortices (surfaces); Source+doublet lines (bodies); Finite-core option

- Discretization - Uniform; Sine; Cosine; Blend

- Control deflections - Via normal-vector tilting; Leading edge or trailing edge flaps; Flaps independent of discretization

- General freestream description - alpha,beta flow angles; p,q,r aircraft rotation components; Subsonic PrandtlGlauert compressibility treatment

- Aerodynamic outputs - Aerodynamic forces and moments, in body or stability axes; Trefftz-plane induced drag analysis; Force and moment derivatives w.r.t. angles, rotations, controls

- Trim calculation:

- Operating variables: alpha, beta; p,q,r; control deflections.

- Constraints: direct constraints on variables; indirect constraints via specified CL, moments

- Multiple trim run cases can be defined, saved, recalled

- Mass properties

- - Optional mass definition file: User-chosen units; Itemized component location, mass, inertias

- - Trim setup of constraints - level or banked horizontal flight; steady pitch rate (looping) flight.

\section{Tornado:}

- Explicit forces in Newton's

- Treffz's plane analysis

- International standard atmosphere

- Velocity in TAS, CAS or Mach

- Compressibility corrections for high subsonic Mach numbers

- Trimmed polars

- Stability derivatives with respect to:

- Pitch and yaw

- Angular rates in pitch, roll and yaw

- Control surface power derivatives

- Parameter sweep

- Dynamic pitch and yaw derivatives.

\section{X-Plane:}

The Blade Element Theory (BET) has shown good results regarding the time of the calculation. In BEM method the blade (or the lifting surface) is divided into several small parts. BEM theory assumes that every one of these smaller sections acts as single two-dimensional airfoil that produces aerodynamic forces and moments. Then these results are integrated to obtain the forces and moments produced by the entire blade (or surface). This method gives sufficiently good results.

\section{OUTPUT DATA}

AVL - the stability derivatives that are output by AVL Editor in an .xml file are shown in Table 1. Each one of them is calculated for several different angles of attack. 
Tornado-output data given by Tornado are shown in Table

Table 1:

\begin{tabular}{|c|c|c|}
\hline$A V L$ & Tornado & Description \\
\hline CDff & - & Trefftz plane induced drag coefficient \\
\hline CDvis & - & Profile drag + CDo drag coefficient \\
\hline $\mathrm{CY}$ & $\mathrm{CC}$ & Side force coefficient \\
\hline $\mathrm{CL}$ & $\mathrm{CL}$ & Lift coefficient \\
\hline $\mathrm{Cl}$ & $\mathrm{Cl}$ & Roll moment coefficient \\
\hline $\mathrm{Cm}$ & $\mathrm{Cm}$ & Pitch moment coefficient \\
\hline $\mathrm{Cn}$ & $\mathrm{Cn}$ & Yaw moment coefficient \\
\hline CLa & $\mathrm{C}_{\mathrm{L} \alpha}$ & Variation of lift coefficient with angle of attack \\
\hline $\mathrm{CLb}$ & $\mathrm{CL}_{\beta}$ & Variation of lift coefficient with sideslip angle \\
\hline $\mathrm{CYb}$ & $\mathrm{CY}_{\beta}$ & Variation of side force coefficient with sideslip angle \\
\hline $\mathrm{Clb}$ & $\mathrm{Cl}_{\beta}$ & Variation of roll moment coefficient with sideslip angle \\
\hline $\mathrm{Cmb}$ & $\mathrm{Cm}_{\beta}$ & Variation of pitch moment coefficient with sideslip angle \\
\hline Cnb & $\mathrm{Cn}_{\beta}$ & Variation of yaw moment coefficient with sideslip angle \\
\hline CLp & CLP & Variation of lift coefficient with roll rate \\
\hline CYp & $\mathrm{CY}_{\mathrm{P}}$ & Variation of side force coefficient with roll rate \\
\hline Clp & Clp & Variation of roll moment coefficient with roll rate \\
\hline Cmp & $\mathrm{Cm}_{\mathrm{P}}$ & Variation of pitch moment coefficient with roll rate \\
\hline Cnp & $\mathrm{Cn}_{\mathrm{P}}$ & Variation of yaw moment coefficient with roll rate \\
\hline $\mathrm{CLq}$ & $\mathrm{CL}_{\mathrm{Q}}$ & Variation of lift coefficient with pitch rate \\
\hline $\mathrm{CYq}$ & $\mathrm{CY}_{\mathrm{Q}}$ & Variation of side force coefficient with pitch rate \\
\hline $\mathrm{Clq}$ & $\mathrm{Cl}_{\mathrm{Q}}$ & Variation of roll moment coefficient with pitch rate \\
\hline $\mathrm{Cmq}$ & $\mathrm{Cm}_{\mathrm{Q}}$ & Variation of pitch moment coefficient with pitch rate \\
\hline Cnq & $\mathrm{Cn}_{\mathrm{Q}}$ & Variation of yaw moment coefficient with pitch rate \\
\hline CLr & $\mathrm{CL}_{\mathrm{R}}$ & Variation of lift coefficient with yaw rate \\
\hline $\mathrm{CYr}$ & $\mathrm{CY}_{\mathrm{R}}$ & Variation of side force coefficient with yaw rate \\
\hline $\mathrm{Clr}$ & $\mathrm{Cl}_{\mathrm{R}}$ & Variation of roll moment coefficient with yaw rate \\
\hline $\mathrm{Cmr}$ & $\mathrm{Cm}_{\mathrm{R}}$ & Variation of pitch moment coefficient with yaw rate \\
\hline Cnr & $\mathrm{Cn}_{\mathrm{R}}$ & Variation of yaw moment coefficient with yaw rate \\
\hline CDffd 1 & & $\begin{array}{l}\text { Variation of induced drag coefficient with control surface } 1 \text { deflection }(\mathrm{deg}) \text { (note } \\
\text { replace } 1 \text { with control surface \# } 1 . . \mathrm{n} \text { for this and all other d1 derivatives) }\end{array}$ \\
\hline CLd1 & & Variation of lift coefficient with control surface 1 deflection (deg) \\
\hline CYd1 & & Variation of side force coefficient with control surface 1 deflection \\
\hline Cld 1 & & Variation of roll moment coefficient with control surface 1 deflection \\
\hline Cmdl & & Variation of pitch moment coefficient with control surface 1 deflection \\
\hline Cndl & & Variation of yaw moment coefficient with control surface 1 deflection \\
\hline
\end{tabular}

X-Plane - The product gives us the opportunity to record a performed in its environment, such as location coordinates; airspeed; aerodynamic forces and moments; accelerations; angular velocities etc. From a dialog window in the product (Fig.1) we can choose which data we want to visualize or wide range of results in a real-time flight simulation export.

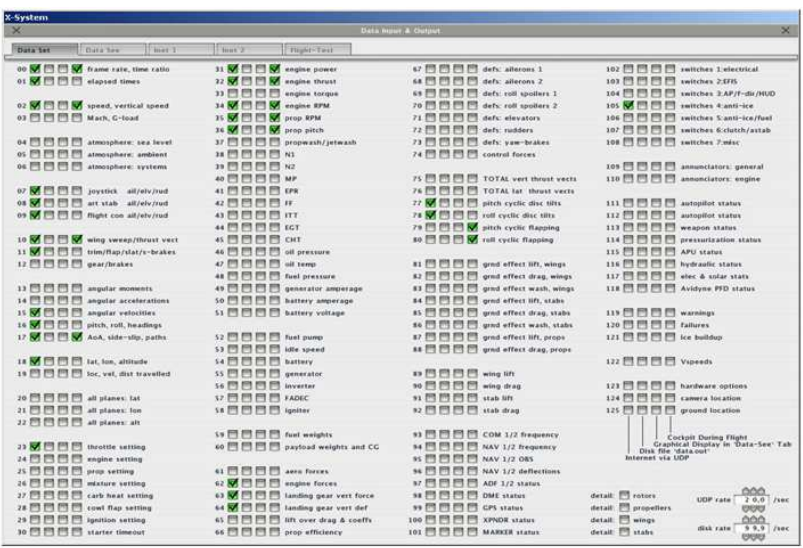

Fig 1 Outputting data in X-Plane

Evaluation of these VLM codes is made (Table 3) based on how close the calculated data is to the data from the experimental flights. A grade from 1 (worst) to 5 (best) is assigned to those stability parameters that can be compared to the source.

\section{COMPARISON OF RESULTS FOR SEVERAL VLM CODES}

In "Surfaces-Vortex-Lattice Module" [7] is presented a comparison table (Table 2) of the results from several different VLM codes and real experimental data for the plane Cessna 172. All stability derivatives are evaluated at $\alpha=0^{0}$.

Table 2

\begin{tabular}{|c|c|c|c|c|c|c|}
\hline & & & & & \multicolumn{2}{|c|}{ Table 2} \\
\hline & 空 & $\sum$ & $\begin{array}{l}\text { 包 } \\
\stackrel{\approx}{=}\end{array}$ & 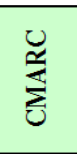 & 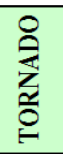 & 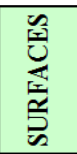 \\
\hline $\mathrm{C}_{\mathrm{L} \alpha}$ & 4,6 & 4,98 & 5,25 & 5,214 & 5,28 & 5,18 \\
\hline $\mathrm{C}_{\mathrm{D} \alpha}$ & 0,13 & 0 & $-0,005$ & 0,086 & -0 & 0,146 \\
\hline $\mathrm{C}_{\mathrm{m} \alpha}$ & $-0,89$ & $-0,33$ & $-0,85$ & $-1,43$ & $-1,5$ & $-1,15$ \\
\hline $\mathrm{C}_{\mathrm{Y} \beta}$ & $-0,3$ & $-0,3$ & $-0,2$ & $-0,1$ & -0 & $-0,3$ \\
\hline $\mathrm{C}_{1 \beta}$ & $-0,09$ & 0,33 & 0,007 & 0,063 & 0,03 & $-0,05$ \\
\hline $\mathrm{C}_{\mathrm{n} \beta}$ & 0,065 & 0,092 & 0,1 & 0,042 & 0,12 & 0,091 \\
\hline $\mathrm{C}_{\mathrm{Yp}}$ & - & $-0,07$ & $-0,1$ & $-0,02$ & -0 & $-0,11$ \\
\hline $\mathrm{C}_{\mathrm{lp}}$ & $-0,47$ & $-0,33$ & $-0,52$ & -1 & $-0,5$ & $-0,51$ \\
\hline $\mathrm{C}_{\mathrm{np}}$ & $-0,03$ & $-0,01$ & $-0,01$ & $-0,13$ & -0 & $-0,02$ \\
\hline $\mathrm{C}_{\mathrm{Lq}}$ & - & 9,41 & 9,3 & 9,003 & 10,2 & 9,111 \\
\hline $\mathrm{C}_{\mathrm{mq}}$ & $-12,4$ & $-14,4$ & -15 & $-17,2$ & -15 & $-12,2$ \\
\hline $\mathrm{C}_{\mathrm{Yr}}$ & 0,21 & 0,209 & 0,23 & 0,45 & 0,27 & 0,306 \\
\hline $\mathrm{C}_{\mathrm{lr}}$ & 0,096 & 0,021 & 0,008 & 0,195 & 0,01 & 0,093 \\
\hline $\mathrm{C}_{\mathrm{nr}}$ & $-0,1$ & $-0,08$ & $-0,095$ & $-0,21$ & $-0,1$ & $-0,12$ \\
\hline
\end{tabular}

Evaluation of these VLM codes is made (Table 3 ) based on how close the calculated data is to the data from the experimental flights. A grade from 1 (worst) to 5 (best) is assigned to those stability parameters that can be compared to the source. 
Table 3

\begin{tabular}{|c|c|c|c|c|c|}
\hline & $\gtreqless$ & 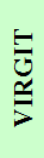 & 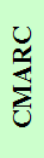 & 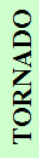 & 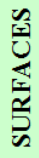 \\
\hline${ }^{\mathrm{C}} \mathrm{L} \alpha$ & 5 & 2 & 3 & 1 & 4 \\
\hline${ }^{\mathrm{C}} \mathrm{D} \alpha$ & 3 & 2 & 5 & 1 & 4 \\
\hline $\mathrm{C}_{\mathrm{m} \alpha}\left(\mathrm{CMy}_{\alpha}\right)$ & 2 & 5 & 3 & 1 & 4 \\
\hline${ }^{\mathrm{c}} \mathrm{Y} \beta$ & 4 & 2 & 1 & 5 & 3 \\
\hline $\mathrm{C}_{1 \beta}\left(\mathrm{CMx}_{\beta}\right)$ & 1 & 4 & 2 & 3 & 5 \\
\hline $\mathrm{C}_{\mathrm{n} \beta}\left(\mathrm{CMz}_{\beta}\right)$ & 4 & 3 & 5 & 1 & 2 \\
\hline $\mathrm{C}_{\mathrm{l}}, \mathrm{P}(\mathrm{CMx}, \mathrm{P})$ & 2 & 4 & 1 & 3 & 5 \\
\hline $\mathrm{C}_{\mathrm{n}}, \mathrm{P}(\mathrm{CMz}, \mathrm{P})$ & 4 & 5 & 1 & 3 & 2 \\
\hline $\mathrm{C}_{\mathrm{m}}, \mathrm{Q}(\mathrm{CMy}, \mathrm{Q})$ & 4 & 2 & 1 & 3 & 5 \\
\hline $\mathrm{C}_{\mathrm{Y}}, \mathrm{R}$ & 5 & 4 & 1 & 3 & 2 \\
\hline $\mathrm{C}_{1}, \mathrm{R}(\mathrm{CMx}, \mathrm{R})$ & 4 & 2 & 1 & 3 & 5 \\
\hline $\mathrm{C}_{\mathrm{n}}, \mathrm{R}(\mathrm{CMz}, \mathrm{R})$ & 2 & 5 & 1 & 4 & 3 \\
\hline TOTAL SCORE & 40 & 40 & 25 & 31 & 44 \\
\hline
\end{tabular}

We can see that AVL scores slightly higher then Tornado in these tests, with only few of the stability parameters shown worse results.

\section{EXPERIMENTAL RESULTS}

As a test case for the products AVL and Tornado we implemented the geometrical model of an experimental UAV JoWi [9] developed at the Technical University Sofia Plovdiv Branch (Figure 2, Figure 3)

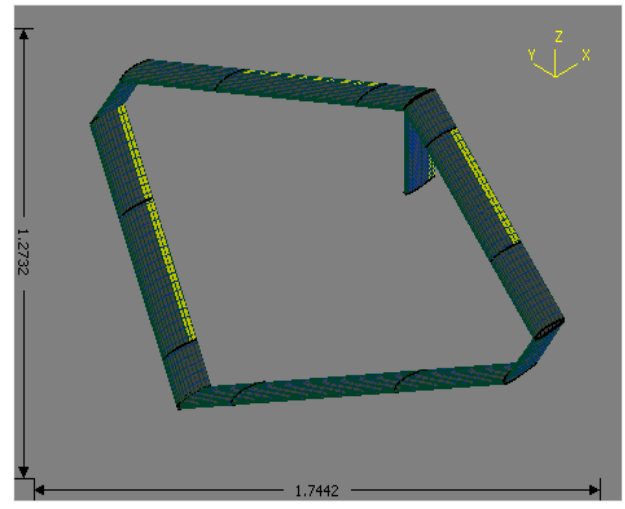

Fig 2 Model of JoWi created in AVL

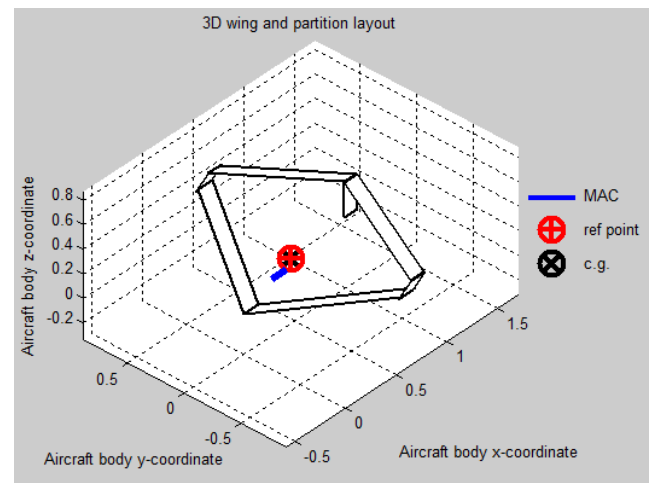

Fig 3 Model of JoWi created in Tornado

Aerodynamic tests were executed and the results for some of the stability derivatives are shown in Table 4:

Table 4

\begin{tabular}{|c|c|c|c|}
\hline & Tornado & AVL & \multirow{9}{*}{$\begin{aligned} \mathrm{V} & =20 \mathrm{~m} / \mathrm{s} \\
\alpha & =6^{\circ} \\
\mathrm{Sfr} & =0,273 \\
\mathrm{~S} & =0,49 \\
\mathrm{Sfr} / \mathrm{S} & =0,5571 \\
\mathrm{~b}_{\mathrm{A}} & =0,145 \\
\text { Stagg } & =0,9\end{aligned}$} \\
\hline $\mathrm{C}_{\mathrm{L}}$ & 0,88073 & 0,63487 & \\
\hline $\mathrm{C}_{\mathrm{D}}$ & 0,03546 & 0,03293 & \\
\hline $\mathrm{C}_{\mathrm{Z}}$ & 0,87961 & $-0,63484$ & \\
\hline $\mathrm{C}_{\mathrm{X}}$ & $-0,05679$ & 0,03362 & \\
\hline $\mathrm{c}_{\mathrm{m}}$ & $-0,13065$ & $-0,03035$ & \\
\hline $\mathrm{C}_{\mathrm{L} \alpha}$ & 4,11873 & 4,14971 & \\
\hline $\mathrm{C}_{\mathrm{Lq}}$ & 0,513 & 1,80402 & \\
\hline $\mathrm{C}_{\mathrm{D} \alpha}$ & 0,3232 & - & \\
\hline $\mathrm{C}_{\mathrm{Dq}}$ & 1,0506 & - & \\
\hline $\mathrm{C}_{\mathrm{Y} \beta}$ & $-0,80524$ & $-0,50767$ & \\
\hline $\mathrm{c}_{1 \beta}$ & 0,07883 & $-0,13539$ & \\
\hline$c_{1 p}$ & $-0,51$ & $-0,48932$ & \\
\hline$c_{1 r}$ & 0,12739 & 0,12755 & \\
\hline $\mathrm{c}_{\mathrm{m} \alpha}$ & $-0,4612$ & $-0,42546$ & \\
\hline $\mathrm{c}_{\mathrm{mq}}$ & $-1,2$ & $-1,4134$ & \\
\hline$c_{n \beta}$ & $-0,09265$ & 0,0453 & \\
\hline$c_{n p}$ & 0,0439 & $-0,07347$ & \\
\hline $\mathrm{c}_{\mathrm{nr}}$ & $-0,0853$ & $-0,05564$ & \\
\hline
\end{tabular}

\section{CONCLUSION}

From the models and calculations that we made with AVL and Tornado we gathered results that give us good overview of the capabilities of these products and their relevancy for the design and research of conceptual UAV's. These products have both their strengths and weaknesses in concrete cases, and for solving specific tasks they can be combined and the results might complement each other. 
One example is that, in order to make the geometric model of the joined wing in Tornado, the wing must be divided into two sections (describing the front and the rear wing), but the product Tornado "recognizes" as main wing only the fist described surface and all the others are treated as secondary (tail surfaces and etc.). In that case the program uses in the calculations only the area (S) of the main wing, which in this specific case (joined wing) requires additional calculations to eliminate the error in data.

One advantage of AVL is the user-friendly interface that the addition to the code - "AVL Editor" offers. It allows us an easy and fast way to model and examine the conceptual plane geometries. On the other side, Tornado can give us visual distribution of the aerodynamic forces and other useful for the research graphics and figures.

We would recommend AVL as the product for aerodynamic research, with the provision to use the other codes as well, when there is a need for additional information, interchangeability or verification of some results, and when it is required or considered appropriate.

\section{REFERENCES}

[1]. Zafirov D., Autonomous VTOL Joined-Wing UAV, AIAA Atmospheric Flight Mechanics (AFM) Conference August 1922, 2013, Boston, MA

[2]. Roglev P., MDO Framework for Conceptual Design of Closed Wing UAV , International Journal of Scientific Research Engineering \& Technology Volume 2 Issue 8 pp 526-531 November 2013

[3]. Panayotov P., Zafirov D., An Ivestigation of the JoinedWing Aerodynamic Characteristics by the VortexLatticeMethod, Sofia, 2003

[4]. Panayotov H., Zafirov D., "Aerodynamic Optimization of Joined-Wing for Unmanned Aerial Vehicle", Journal of the Technical University at Plovdiv Fundamental Sciences and Applications Vol. 13 (8), Plovdiv, Bulgaria, 2006.

[5]. http://web.mit.edu/drela/Public/web/avl/ - official website of the product AVL

[6]. http://www.redhammer.se/tornado/ - official website of the product Tornado

[7]. Surfaces - Vortex-Lattice Module, Great OWL Publishing, 2009

[8]. Tomas Melin, A Vortex Lattice MATLAB Implementation for Linear Aerodynamic Wing Applications, Royal Institute of Technology, 2000

[9]. Zafirov D., Autonomous VTOL Joined-Wing UAV, AFM

Conference, AIAA, Boston 2013

\section{BIOGRAPHIES}

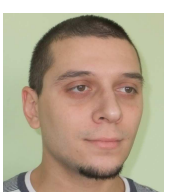

Jordan Hadjiev is doctoral students in the field of UAVs. Her interests are focused on the study of joined wing aircraft.

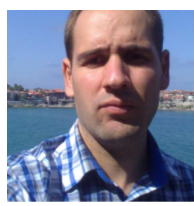

Hristian Panayotov is an associated professor in the Technical University of Sofia. He is working in the area of UAV design, aerodynamics and optimization. $\mathrm{He}$ is an author of numerous publications on joinedwing aerodynamics and design. 